\title{
Profilaxis antibiótica en el manejo conservador de los pacientes con trauma renal: ¿Aún existe controversia?
}

\section{Antibiotic Prophylaxis in Conservative Management of Patients with Renal Trauma: Is it still Controversial?}

\author{
Herney Andrés García-Perdomo ${ }^{1}$ Diego Andrés Gómez-Puerto ${ }^{2} \quad$ James Alejandro Zapata-Copete 3 \\ Ramiro Manzano-Núñez ${ }^{4}$ \\ 1 Profesor Asociado Universidad del Valle. Director Grupo de \\ Investigación UROGIV y Grupo Asociado Cochrane \\ 2 Universidad del Valle, Cali, Colombia \\ ${ }^{3}$ Universidad Libre - Grupo de Investigación UROGIV. Universidad del \\ Address for correspondence Herney García, MD, MSc, EdD, PhD, \\ FACS, Dirección Cll 4B \#36-00, Cali- Colombia \\ Valle, Cali, Colombia \\ ${ }^{4}$ Fundación Valle del Lili - Grupo de Investigación UROGIV. \\ Universidad del Valle, Cali, Colombia \\ Urol Colomb 2019;28:221-225.
}

\section{Resumen}

\section{Palabras Clave}

- heridas y lesiones

- lesiones abdominales

- riñón

- profilaxis antibiótica
Objetivo Describir la evidencia que existe en la actualidad acerca del uso del antibiótico profiláctico en el manejo conservador del trauma renal.

Material y métodos Decidimos realizar una revisión de la literatura para intentar resolver la controversia existente acerca de la efectividad del uso de antibiótico profiláctico en pacientes con trauma renal para prevenir complicaciones infecciosas. Resultados El trauma renal es un problema importante en los servicios de urgencias en el mundo. La mayoría corresponden a traumatismos cerrados y principalmente a accidentes de tránsito, aunque pueden ser secundarios a un trauma penetrante. Los abscesos perirrenales son poco frecuentes, se estima que ocurren en menos del $1 \% \mathrm{de}$ todos los casos de trauma renal y en aproximadamente $5 \%$ de los casos de trauma renal penetrante. Dada la baja incidencia de abscesos perirrenales y las diferentes corrientes existe controversia para el tratamiento antibiótico. El manejo es conservador en la mayoría de los casos, y depende del grado de la lesión, el compromiso vascular y el estado hemodinámico del paciente.

Conclusión Aún no existe claridad acerca de la utilización de antibiótico profiláctico en el manejo conservador del paciente con un traumatismo renal, relacionado con el tipo de trauma y el grado de la lesión. Sugerimos el uso de antibiótico profiláctico en pacientes con trauma penetrante grado IV dada la mezcla de ambiente externo, cuerpo extraño y orina, sin embargo, no hay evidencia de alta calidad al respecto.

Objective To describe the current evidence on the use of the prophylactic antibiotic in the conservative management of renal trauma. received

July 11,2017

accepted

October 11, 2017
DOI https://doi.org/

$10.1055 / \mathrm{s}-0038-1651510$.

ISSN 0120-789X.

eISSN 2027-0119.
Copyright $($ C 2019, Sociedad Colombiana License terms de Urología. Publicado por Thieme Revinter Publicações Ltda., Rio de Janeiro, Brazil. Todos los derechos reservados.

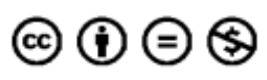




\section{Keywords}

- wounds and injury

- abdominal injuries

- kidney

- wounds

- antibiotic prophylaxis
Materials and Methods We decided to review the general and updated literature to try to solve the controversy about the effectiveness of the use of antibiotic prophylaxis in patients with renal trauma to prevent infectious complications.

Results Renal trauma is an important trauma in emergency services around the world. Most of them are blunt and mainly because of transit accidents, although they could be due to penetrating trauma. Perirenal abscesses are uncommon, it is estimated that occur in less than $1 \%$ of all cases of renal trauma and $~ 5 \%$ of cases of penetrating renal trauma. Given the low incidence of perirenal abscesses and different streams, controversy exists for antibiotic treatment. Conservative management is the preferred choice, normally according to the grade of the trauma, the vascular compromise and the patients hemodynamic response.

Conclusion There is still no consensus about the use of prophylaxis antibiotics in conservative management of patients with renal trauma, related to type and the grade of trauma. We suggest the use of a prophylactic antibiotic in patients with grade IV penetrating trauma given the mixture of external environment, foreign body and urine, however there is no evidence of high quality on this regard.

\section{Introducción}

El trauma renal representa aproximadamente entre un $8 \%$ a un $10 \%$ de los casos de trauma de abdomen y corresponde entre el $1 \%$ y el $5 \%$ de todos los casos de trauma. ${ }^{1}$ Se estima que entre el $80 \%-90 \%$ de los casos son secundarios a un traumatismo cerrado y un $10 \%-20 \%$ a trauma penetrante. Los mecanismos de trauma incluyen accidentes de tránsito (aproximadamente 50\%), caídas (16\%), deportes (golpe directo en el flanco o abdomen) y asalto. La desaceleración súbita o lesiones por trauma contuso son las que frecuentemente pueden dar lugar a una contusión renal o a una laceración del parénquima y/o del sistema colector. Muy raramente se produce una lesión vascular. ${ }^{2}$ La mayoría de los pacientes son tratados en forma conservadora y tienen una recuperación completa, teniendo un pronóstico global dependiente del tipo de lesiones, teniendo mejor pronóstico las lesiones menores (Grado I - III). La profilaxis antibiótica en el manejo conservador del trauma renal sigue siendo controvertida, principalmente en los de alto grado. El objetivo del presente trabajo fue describir la evidencia que existe en la actualidad acerca del uso del antibiótico profiláctico en el manejo conservador del trauma renal.

\section{Materiales y métodos}

Se realizó una búsqueda de literatura en bases de datos en Medline a través de Ovid, Scopus (incluido Embase) y LILACS desde 1980 hasta la actualidad. Se utilizaron los términos MeSH, Emtree y DeCS, así como el lenguaje no controlado. Las palabras genéricas usadas fueron: heridas y lesiones; trauma renal; profilaxis antibiótica; heridas/ trauma abdominal. Fueron seleccionados los artículos relevantes para la estructura y desarrollo del presente trabajo.

\section{Clasificación del trauma renal y principios del manejo conservador}

Las lesiones renales se pueden clasificar según su mecanismo, como penetrantes o no penetrantes (cerradas). En las zonas rurales el traumatismo cerrado comprende el mayor porcentaje de lesiones, correspondiendo a un $90 \%-$ 95\%; mientras que en el medio urbano las lesiones penetrantes comprenden casi un $40 \%$ del total y por lo general son secundarias a heridas por arma de fuego y heridas por arma blanca. Los mecanismos en el trauma no penetrante incluyen colisiones de vehículos, caídas, accidentes de peatones asociados al vehículo entre otros; siendo los accidentes de tráfico los causantes de casi la mitad de todas las lesiones cerradas. ${ }^{3}$ Por otro lado, aunque menos frecuentes, los traumatismos penetrantes tienden a generar lesiones más graves que las del traumatismo cerrado. ${ }^{4}$

La clasificación comúnmente utilizada es la de la Asociación Americana para la Cirugía de Trauma (AAST) (-Tabla 1). Ésa ha sido validada ayudando a predecir la necesidad de intervención, la morbilidad para la lesión renal, y la mortalidad en lesiones no penetrantes. ${ }^{5-7}$

La mayoría de los pacientes con lesiones renales significativas requieren atención en urgencias con evaluación urológica especializada. El componente fundamental del tratamiento conservador consiste en el reposo absoluto hasta la resolución de la hematuria, además de una evaluación y observación continúa con estimaciones seriadas del hematocrito. ${ }^{8}$ La necesidad de exploración renal puede ser establecida por el grado de inestabilidad hemodinámica, la necesidad de transfusión, el valor de creatinina y el grado de la lesión. ${ }^{9}$ Las contraindicaciones absolutas para la realización del tratamiento conservador incluyen hipotensión no controlada, sangrado activo, avulsión del pedículo renal y la presencia de un hematoma pulsátil y/o expansible. 
Tabla 1 Clasificación de lesiones por trauma renal según $\mathrm{AAST}^{6,7}$

\begin{tabular}{|l|l|}
\hline Grado* $^{*}$ & Descripción de la lesión \\
\hline I & $\begin{array}{l}\text { Contusión o hematoma subcapsular no } \\
\text { expansible }\end{array}$ \\
\hline & No laceración \\
\hline II & Hematoma perirenal no expansible \\
\hline III & $\begin{array}{l}\text { Laceración cortical }<1 \mathrm{~cm} \text { de profundidad sin } \\
\text { extravasación }\end{array}$ \\
\hline IV & $\begin{array}{l}\text { Laceración cortical }>1 \mathrm{~cm} \text { sin extravasación } \\
\text { urinaria }\end{array}$ \\
\hline & $\begin{array}{l}\text { Laceración: A través de la unión corticomedular } \\
\text { hacia el sistema colector }\end{array}$ \\
\hline & Lesión vascular parcial o trombosis vascular \\
\hline V & $\begin{array}{l}\text { Vascular: Arteria renal segmentaria o lesión } \\
\text { venosa con hematoma contenido }\end{array}$ \\
\hline & Laceración: Riñón Destruido \\
\hline
\end{tabular}

*Avanzar un grado para lesiones bilaterales de hasta grado III.

Las lesiones grado I son las más frecuentes (75\%-85\% de los casos), se caracterizan por pequeñas contusiones y hematomas subcapsulares pequeños y no expansivos, y se manejan de manera conservadora. ${ }^{10,11}$

Las lesiones de grado II, se caracterizan por hematomas perinéfricos no expansivos confinados al retroperitoneo y/o por laceraciones corticales superficiales de menos de $1 \mathrm{~cm}$ de profundidad sin daño al sistema colector. Ese tipo de lesiones se pueden manejar de manera conservadora ya que se ha demostrado que ese manejo tiene un éxito de hasta el $95 \%{ }^{11,12}$ La diferencia entre las lesiones de grado II y grado III es la profundidad de la laceración renal. Las lesiones de grado III de caracterizan por laceraciones $>1 \mathrm{~cm}$ sin compromiso del sistema colector. Las lesiones de grado III pueden manejarse de manera conservadora en pacientes estables en más del $90 \%$ de los casos. ${ }^{8,11}$

Las lesiones grado IV se caracterizan por laceraciones profundas del parénquima renal que se extienden a través de la corteza y médula renal hasta el sistema colector y por lesiones que comprometen la arteria o venal renal causando hemorragia contenida e infartos segmentarios sin laceraciones asociadas. El manejo de ese tipo de lesiones es complejo y no hay consenso sobre cuál es la mejor aproximación en esos pacientes; sin embargo, algunos casos pueden ser manejados de manera conservadora con el riesgo que requieran una intervención quirúrgica de urgencia o un reparo posterior. ${ }^{13,14}$

Las lesiones de grado $\mathrm{V}$ incluyen laceraciones múltiples del parénquima renal y lesiones vasculares que comprometen el pedículo vascular. En ese tipo de lesiones, el manejo conservador y quirúrgico han sido reportados con resultados heterogéneos, ${ }^{11-13,15}$ por lo cual el abordaje es mucho más controversial. Usualmente las lesiones de grado $\mathrm{V}$ son indicación de exploración quirúrgica, pero pacientes estables y con daño solamente del parénquima renal pueden ser manejados de manera conservadora. ${ }^{13,16,17}$
Las lesiones penetrantes generan controversia acerca de la necesidad de intervención quirúrgica, principalmente cuando vienen acompañadas de otros factores como lesiones renovasculares, hematoma retroperitoneal no pulsátil, extravasación urinaria y lesiones en otros órganos. ${ }^{3,10}$

El manejo conservador está recomendado en todos los pacientes con lesiones de Grado I a III, sean penetrantes o no, siempre y cuando haya estabilidad hemodinámica. ${ }^{11,14}$ Las lesiones de grado IV y $\mathrm{V}$ tienen mayor posibilidad de complicaciones y según el tipo de lesión se tendrá en cuenta el tipo de intervención, sin embargo, aún no existe consenso en la literatura.

Si el paciente se encuentra inestable hemodinámicamente, se debe sospechar de una lesión vascular y se debe evaluar la posibilidad de embolización o cirugía para exploración renal (de acuerdo con la disponibilidad del recurso). Si hay urinoma, se debe considerar la posibilidad de colocar un stent ureteral o realizar una exploración renal por vía abierta o laparoscópica, individualizando cada paciente. ${ }^{11}$

\section{Riesgo de complicaciones infecciosas y uso de antibióticos. ¿Cuándo y cómo usarlos?}

El trauma renal trae consigo el riesgo de complicaciones infecciosas posteriores como abscesos perirrenales $\mathrm{y}$ urinomas infectados. La mayoría, secundarios a la contaminación bacteriana proveniente de diferentes vías; hematógena, retrógrada, por lesión externa o por vecindad con los diferentes órganos, predominantemente el intestino. ${ }^{8}$

Los abscesos perirrenales son poco frecuentes, se estima que ocurren en menos del $1 \%$ de todos los casos de trauma renal $^{15,18}$ y en aproximadamente $5 \%$ de los casos de trauma renal penetrante. ${ }^{19}$ Los abscesos deben sospecharse en pacientes que desarrollan fiebre luego de un trauma renal con un urinoma o un hematoma perinéfrico y son más frecuentes cuando se presentan factores predisponentes. La presencia de segmentos renales desvitalizados, pérdida significativa de tejidos blandos y lesiones concomitantes intestinales o pancreáticas aparentemente aumenta el riesgo de infección. ${ }^{16,20,21}$ Otros factores como la diabetes y la infección por Virus de Inmunodeficiencia Humana (HIV), podrían incrementar el riesgo del absceso perinéfrico en esos pacientes sin embargo, no existe evidencia al respecto. Es importante anotar que aunque el riesgo de infección en trauma cerrado es casi nulo debido a que se trata de una lesión no contaminada, teóricamente él aumentaría en casos en los que haya extravasación de la orina asociada a la presencia de catéteres intravasculares y urinarios, ya que esos dos factores podrían favorecer la colonización bacteriana. ${ }^{10,16}$ Así pues, existe controversia en el uso de antibióticos profilácticos en trauma renal. Se ha sugerido que pacientes con lesiones de grado IV y $\mathrm{V}$ podrían recibir antibiótico endovenoso por al menos 48-72 horas seguido por un curso de 5 días más por vía oral. ${ }^{11,22}$ Sin embargo, no existe evidencia que apoye dicha conducta, por lo cual se podría sugerir que dado el bajo riesgo de infección (menor al $5 \%$ ), no estaría indicada la profilaxis en pacientes con manejo conservador de trauma renal. Por otro lado, otros autores consideran que la administración de antibióticos podría 
plantearse en pacientes con trauma penetrante grado IV en los que hay lesiones en contacto con el medio externo y extravasación urinaria, donde el uso de antibióticos podría ser una intervención eficiente. ${ }^{8}$ No obstante, tampoco existe evidencia de alta calidad al respecto y se requieren estudios complementarios para ofrecer una clara recomendación.

Adicionalmente, en concordancia con la esencia del manejo conservador del trauma renal, pacientes con trauma menor (Grado I - III), contuso o penetrante, con estabilidad hemodinámica y sin factores de riesgo, no se deben administrar antibióticos de manera profiláctica. ${ }^{23}$

El uso de antibióticos profilácticos fuera del escenario mencionado queda a criterio clínico según los factores de riesgo con los que se presente el paciente. Es importante anotar que en presencia de fiebre, lesiones de cualquier grado deben recibir antibioticoterapia. ${ }^{11}$

Por último, la elección del tipo de antibiótico debe ser determinada por los posibles microorganismos contaminantes que podrían causar infección. Se pueden utilizar cefalosporinas de primera generación por su acción contra estafilococos, cobertura razonable contra grampositivos y bajo riesgo de selección de organismos multirresistentes si se usan durante corta duración. ${ }^{11}$ También se recomienda el uso de antibióticos endovenosos de amplio espectro si hay posibilidad de daños en el sistema colector y fuga de orina, ${ }^{24}$ sin embargo, no existe evidencia clara que soporte el uso de un agente específico. Otras opciones alternativas incluyen ciprofloxacina o tratamiento combinado con ampicilina y gentamicina. Cualquier lesión intestinal concomitante requiere cobertura anaeróbica adicional en forma de metronidazol o clindamicina. ${ }^{11}$

\section{Conclusión}

El uso de antibióticos profilácticos se realiza en la práctica clínica sin límites claros por la falta de evidencia acerca de las indicaciones. Existe controversia en cuanto al tipo de traumas que requieren profilaxis antibiótica, sugerimos el uso de antibiótico profiláctico en pacientes con trauma penetrante grado IV dada la mezcla de ambiente externo, cuerpo extraño y orina, sin embargo, no hay evidencia de alta calidad al respecto y se requieren estudios complementarios para ofrecer una clara recomendación.

Por los pocos estudios y evidencia disponible, no es posible llevar a cabo una revisión sistemática. Sugerimos la realización de estudios que comparen la profilaxis antibiótica con el manejo usual.

\section{Declaración}

Los autores declaran que no hay conflictos de interés ni recibieron fondos para el desarrollo del presente trabajo.

\section{Contribuciones de los autores}

Los autores arriba mencionados, declaran que han contribuido de manera significativa en la concepción, construcción, finalización y aprobación del manuscrito final para ser enviado a Urología Colombiana.
Declaración de conflicto de intereses

Los autores arriba mencionados, declaran que no tienen conflicto de ningún tipo relacionado con la elaboración de este artículo. No hay nada que declarar.

\section{Referencias}

1 Patel P. Management of renal injuries in blunt abdominal trauma. Duttaroy D, editor. J Res Med Dent Sci. 2014;2(2):38-42

2 Lumen N, Kuehhas FE, Djakovic N, y col. Review of the current management of lower urinary tract injuries by the EAU Trauma Guidelines Panel. Eur Urol 2015;67(05):925-929

3 Santucci RA, Wessells H, Bartsch G, y col. Evaluation and management of renal injuries: consensus statement of the renal trauma subcommittee. BJU Int 2004;93(07):937-954

4 Makanjuola JK, Grange PCR, Kooiman G, Brown C, Sharma DM. Centralisation of major trauma: an opportunity for acute urology services in the UK. BJU Int 2012;109(02):173-174

5 Kuan JK, Wright JL, Nathens AB, Rivara FP, Wessells H; American Association for the Surgery of Trauma. American Association for the Surgery of Trauma Organ Injury Scale for kidney injuries predicts nephrectomy, dialysis, and death in patients with blunt injury and nephrectomy for penetrating injuries. J Trauma 2006; 60(02):351-356

6 Santucci RA, McAninch JW, Safir M, Mario LA, Service S, Segal MR. Validation of the American Association for the Surgery of Trauma organ injury severity scale for the kidney. J Trauma 2001;50(02): 195-200

7 Moore EE, Shackford SR, Pachter HL, y col. Organ injury scaling: spleen, liver, and kidney. J Trauma 1989;29(12):1664-1666

8 Shoobridge JJ, Corcoran NM, Martin KA, Koukounaras J, Royce PL, Bultitude MF. Contemporary management of renal trauma. Rev Urol 2011;13(02):65-72

9 Shariat SF, Trinh Q-D, Morey AF, y col. Development of a highly accurate nomogram for prediction of the need for exploration in patients with renal trauma. J Trauma 2008;64(06):1451-1458

10 Heyns CF. Renal trauma: indications for imaging and surgical exploration. BJU Int 2004;93(08):1165-1170

11 McCombie SP, Thyer I, Corcoran NM, y col. The conservative management of renal trauma: a literature review and practical clinical guideline from Australia and New Zealand. BJU Int 2014; 114(Suppl 1):13-21

12 Yang C-S, Chen IC-J, Wang C-Y, Liu C-C, Shih H-C, Huang M-S. Predictive indications of operation and mortality following renal trauma. J Chin Med Assoc 2012;75(01):21-24

13 van der Wilden GM, Velmahos GC, Joseph DK, y col; GM van der W. Successful nonoperative management of the most severe blunt renal injuries: a multicenter study of the research consortium of New England Centers for Trauma. JAMA Surg 2013;148(10): 924-931

14 Long J-A, Fiard G, Descotes J-L, y col. High-grade renal injury: nonoperative management of urinary extravasation and prediction of long-term outcomes. BJU Int 2013;111(4 Pt B):E249-E255

15 Dobrowolski Z, Kusionowicz J, Drewniak T, y col. Renal and ureteric trauma: diagnosis and management in Poland. BJU Int 2002;89(07):748-751

16 Moudouni SM, Patard JJ, Manunta A, Guiraud P, Guille F, Lobel B. A conservative approach to major blunt renal lacerations with urinary extravasation and devitalized renal segments. BJU Int 2001;87(04):290-294

17 García HA, Urrea MF, Serna A, Aluma LJ. Experiencia clínica en el manejo del traumatismo renal en el Hospital Universitario del Valle (Cali, Colombia). Actas Urol Esp 2009;33(08):881-887

18 Kansas BT, Eddy MJ, Mydlo JH, Uzzo RG. Incidence and management of penetrating renal trauma in patients with 
multiorgan injury: extended experience at an inner city trauma center. J Urol 2004;172(4 Pt 1):1355-1360

19 Wilson RF, Ziegler DW. Diagnostic and treatment problems in renal injuries. Am Surg 1987;53(07):399-402

20 Husmann DA, Gilling PJ, Perry MO, Morris JS, Boone TB. Major renal lacerations with a devitalized fragment following blunt abdominal trauma: a comparison between nonoperative (expectant) versus surgical management. J Urol 1993;150(06):1774-1777

21 Rosen MA, McAninch JW. Management of combined renal and pancreatic trauma. J Urol 1994;152(01):22-25
22 McGuire J, Bultitude MF, Davis P, Koukounaras J, Royce PL, Corcoran NM. Predictors of outcome for blunt high grade renal injury treated with conservative intent. J Urol 2011;185(01): 187-191

23 Broghammer JA, Fisher MB, Santucci RA. Conservative management of renal trauma: a review. Urology 2007;70(04): 623-629

24 da Costa IA, Amend B, Stenzl A, Bedke J. Contemporary management of acute kidney trauma. J Acute Dis 2015;5(01): 29-36 